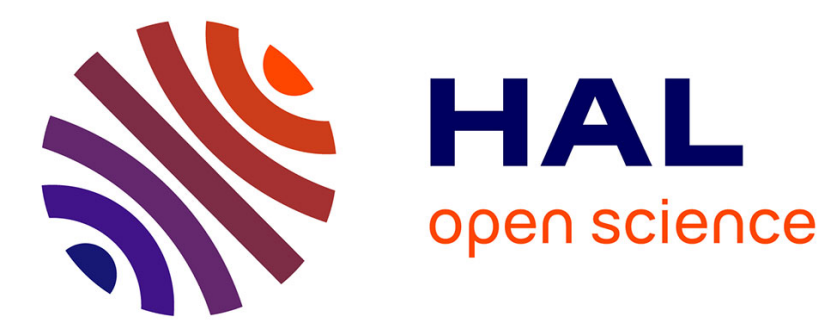

\title{
Stress, risque suicidaire et annonce de son homosexualité
}

Elodie Charbonnier, Pierluigi Graziani

\section{To cite this version:}

Elodie Charbonnier, Pierluigi Graziani. Stress, risque suicidaire et annonce de son homosexualité.

Service Social, 2013, 59 (1), pp.1-16. hal-01623616

\section{HAL Id: hal-01623616 \\ https://hal.science/hal-01623616}

Submitted on 12 Jan 2018

HAL is a multi-disciplinary open access archive for the deposit and dissemination of scientific research documents, whether they are published or not. The documents may come from teaching and research institutions in France or abroad, or from public or private research centers.
L'archive ouverte pluridisciplinaire $\mathbf{H A L}$, est destinée au dépôt et à la diffusion de documents scientifiques de niveau recherche, publiés ou non, émanant des établissements d'enseignement et de recherche français ou étrangers, des laboratoires publics ou privés. 
Auteurs : Élodie Charbonnier et Pierluigi Graziani

Élodie Charbonnier, psychologue clinicienne, doctorante en psychologie clinique et psychopathologie. Unîmes, 30021 Nîmes, France et Université d'Aix-Marseille, 13100, Aixen-Provence, France. LPS EA 849.

Pierluigi Graziani, professeur de psychologie clinique et psychopathologie, Unîmes 30021, Nîmes, France. LPS EA 849.

Cette recherche bénéficie du soutien du Groupement d'Étude et de Prévention du Suicide (GEPS), et d'une subvention de la Fondation Pfizer, pour la santé de l'enfant et de l'adolescent.

La correspondance concernant cet article est à adresser à Élodie Charbonnier.

Courriel : charbonnier.elodie@gmail.com 


\title{
Résumé :
}

Cette recherche vise à mieux comprendre le risque suicidaire élevé des jeunes lesbiennes et gays. Pour cela, nous avons évalué les liens pouvant exister entre leur risque suicidaire et l'important stress qu'elles et ils ressentent lors de la divulgation de leur homosexualité. Des entretiens semi-directifs ont été réalisés auprès de 15 jeunes se disant lesbiennes ou gays. Au sein de notre échantillon, trois jeunes avaient fait une tentative de suicide, dont deux en lien avec l'annonce de leur homosexualité. Nos résultats démontrent que cette divulgation peutêtre génératrice d'une importante détresse et conduire certains jeunes à avoir envie de mettre fin à leurs jours, essentiellement lorsqu'elle donne lieu à des attitudes négatives. Mais elle peut aussi être source de libération et de bien-être, notamment lorsqu'elle conduit le jeune à bénéficier d'un soutien plus important.

Mots clés : suicide, annonce de son homosexualité, émotions, stress

\begin{abstract}
:
This research aims at understanding the high suicidal risk of the young lesbians and gays. For that purpose, we evaluated potentials connections between their suicidal risk and the important stress they felt during the coming out. Semi-directive interviews were realized with 15 young people considering thremselves lesbians or gays. Within our sample, three young people had made a suicide attempt, among which two in connection with their coming out. Our results demonstrate that this disclosure may generate important distress and lead some young people trying to take off their life, essentially when it rises negative attitudes. But it can also cause well-being and relief, primarily when it leads the young person to benefit a more important support.
\end{abstract}

Keywords: suicide, disclosure your homosexuality, emotions, stress 
Stress, risque suicidaire et annonce de son homosexualité

Cette recherche qualitative est exploratoire, elle se base sur le discours de 15 jeunes (1828 ans) se disant lesbiennes ou gays (LG). Elle a pour objectif de mieux comprendre le stress que ces jeunes ont ressenti lors de la divulgation de leur homosexualité la plus stressante de leur vie. Notre second objectif est d'appréhender les contextes dans lesquels elles et ils ont présenté des idéations et des conduites suicidaires. Cette recherche s'appuie sur le « modèle transactionnel du stress » (Lazarus, 1966 ; Coyne et Lazarus, 1980 ; Lazarus et Folkman, 1984) et sur le minority stress model (Meyer, 2003 ; Meyer et Northridge, 2007 ; Meyer, Dietrich et al., 2008).

\section{Risque suicidaire et homosexualité}

Depuis les années 1980, les recherches étasuniennes rendent compte de la sur-suicidabilité des jeunes lesbiennes, gays et bisexuels (LGB), plus particulièrement des hommes (Mathy, 2002 ; Remafedi, 2002). Selon le rapport du Suicide Prevention Resource Center les jeunes LGB ont entre 1,5 et 3 fois plus de risque d'avoir des idées suicidaires que leurs pairs hétérosexuels (Suicide Prevention Resource Center, 2008). Par exemple, dans l'étude menée par Silenzo et ses collaborateurs, $17,2 \%$ des 447 jeunes LGB (18-26 ans) interrogés ont déclaré avoir eu des idées suicidaires durant les douze derniers mois contre $6,3 \%$ des 13885 hétérosexuels du même âge (Silenzio, Peña et al., 2007). Les données françaises vont dans le même sens. Une des premières recherches françaises (l'enquête "Presse Gay») a été menée par voie de presse. Cette enquête a été réalisée auprès de 4753 gays, dont $17 \%$ ont déclaré avoir déjà fait une tentative de suicide au cours de leur vie, le taux étant de $27 \%$ pour les moins de 20 ans (Adam, 2001). Cette enquête a été reconduite en 2004 auprès de 6184 gays ; le pourcentage de jeunes hommes ayant fait une tentative de suicide atteignait alors $19 \%$ (et $32 \%$ pour les 
moins de 20 ans) (Velter, 2007). De même, le Baromètre Santé 2005 mené auprès de 16883 sujets (15-75 ans) atteste d'une prévalence des tentatives de suicide au cours de la vie plus importante chez les jeunes hommes gays et bisexuels que chez les hétérosexuels $(7,5 \%$ vs 2,5\%) (Jouvin, Beaulieu-Prévost et al., 2007). Cette sur-suicidabilité a été confirmée par une recherche française plus récente (Bourdet et Pugnière, 2011). Afin de compléter ces données quantitatives, Verdier et Firdion (2003) ont interrogé 13 personnes LGB qui ont fait une ou plusieurs tentatives de suicide afin de comprendre les facteurs impliqués dans leurs passages à l'acte. Tous font état d'un mal-être identitaire, d'un «sentiment de ne pas être normal », la « honte d'être soi » étant une dimension commune à tous les répondants (Verdier et Firdion, 2003).

Les résultats des enquêtes et études canadiennes confirment les données françaises et étasuniennes. En effet, les recherches canadiennes rendent compte d'une prévalence plus marquée des idéations et des tentatives de suicide chez les LGB que chez les hétérosexuels (Bagley et Tremblay, 1997 ; Clermont et Lacouture, 2000 ; Otis, Noël et al., 2002). De même, « le plan d'action en santé mentale 2005-2010 » mené au Québec précise que les personnes vivant une crise liée à leur orientation sexuelle ont plus de risque de commettre une ou des tentatives de suicide (ministère de la Santé et des Services sociaux, 2010). Au-delà des conduites suicidaires, les données canadiennes mettent également en lumière la prévalence de nombreux signes de souffrance (niveau de détresse émotionnelle, d'anxiété et de dépression) chez les LGB comparativement aux hétérosexuels (Saewyc, Skay et al., 2007). Tout comme Verdier et Firdion (2003) en France, Dorais et Lajeunesse (2001) au Canada ont pris le parti de s'intéresser aux récits de vie des jeunes gays ayant fait au moins une tentative de suicide. Ces données leur ont permis de dégager quatre scénarios possibles. Le « parfait garçon », qui en devenant perfectionniste cherche à se dédouaner de sa différence. Le "caméléon », qui 
« cache » son homosexualité et qui se perçoit plus ou moins comme un imposteur. Le « fif de service » est le bouc émissaire des autres, identifié très tôt comme homosexuel. Le « rebelle » quant à lui adopte une posture de révolte et d'opposition, notamment contre l'homophobie. Ces auteurs précisent que c'est dans la famille et à l'école que les difficultés de ces jeunes au cours de leur adolescence sont les plus grandes. Ainsi, les études quantitatives permettent de quantifier la prévalence des conduites et idéations suicidaires des jeunes LGB, mais la démarche qualitative apparait être un complément essentiel afin mieux comprendre leur risque suicidaire. Par conséquent, nous avons fait le choix de privilégier une méthodologie qualitative pour notre recherche.

\section{Stress et annonce de son homosexualité}

Selon le minority stress model (MSM), ces taux élevés d'idéations et de conduites suicidaires seraient liés aux différents stresseurs vécus par les LGB (Meyer, 2003). Le MSM défend l'idée que les personnes appartenant à une minorité présenteraient des stresseurs spécifiques (comme par exemple pour les LGB l'annonce de leur homosexualité ou encore les propos homophobes), qui s'additionneraient aux stresseurs de la population générale et rendraient ces personnes plus vulnérables, ce qui augmenterait leur risque suicidaire lors des transactions stressantes (Meyer, 1995 ; Meyer, 2003 ; Meyer et Northridge, 2007). Ces stresseurs spécifiques sont regroupés sous le terme minority stress. En effet, la période du coming-out durant laquelle les LGB essaient d'accepter leur orientation sexuelle et la divulgue à leur entourage est particulièrement à risque (Paul, Catania et al., 2002). Ce processus peut être difficile lorsque les individus sont envahis par de nombreuses craintes, notamment concernant les réactions négatives de leur entourage, qu'elles soient réelles ou anticipées (ministère de la Santé et des Services sociaux, 1997). En effet, la plupart des tentatives de suicide se 
produiraient à un moment où le jeune craint de faire son coming-out ou vient tout juste de le faire (Dorais et Lajeunesse, 2001).

L'annonce de son homosexualité, spécifique aux LGB, est fréquemment une expérience stressante (Wong et Tang, 2004) durant laquelle la personne se définit comme différente (Bonet, Wells et al., 2007) et qui implique la possibilité d'un rejet de la part des personnes destinataires de l'annonce (D’Augelli, 2002 ; Meyer, 2003). Parmi les 1906 jeunes LGB (1217 ans) interrogés dans la recherche de Padilla et de ses collaborateurs (2010), $58 \%$ pensent que leur orientation sexuelle constituera un obstacle dans leur vie, et ce, de manière plus marquée chez les hommes. Les LGB sont donc nombreux à ne pas annoncer leur homosexualité à leurs parents ou à leur entourage (D’Augelli, Grossman et al., 2010). L'annonce à la mère se fait en général avant la divulgation au père, ce qui serait le reflet d'une plus grande confiance, d'une plus grande proximité émotionnelle et d'une relation plus proche vis-à-vis de celle-ci (Savin-Williams, 2001). De plus, la réaction maternelle évolue également plus positivement que la paternelle (Charbonnier et Graziani, 2011). Les croyances religieuses constituent une des principales barrières à cette divulgation (Padilla, Crisp et al., 2010). Bien que le dévoilement de son homosexualité soit en général considéré comme un moment difficile et stressant (Savin-Williams, 2001; Willoughby, Malik et al., 2006), il peut aussi avoir des effets positifs (Iwasaki et Ristock, 2007). On observe par exemple chez de nombreux LGB une atténuation de la dépression et de l'anxiété (LaSala, 2000 ; Stevens, 2004), plus de bien-être, une amélioration de la qualité de vie (Savin-Williams, 2001 ; Halpin et Allen, 2004), des émotions de soulagement (Monroe, 2001 ; Charbonnier et Graziani, 2012), une transformation positive de l'identité personnelle et sociale (Morris, 1997) et une meilleure acceptation de son orientation sexuelle (Rosario, Schrimshaw et al., 2004). 
L'annonce de son homosexualité est donc un stresseur spécifique aux LGB (minority stress) qui est complexe et qui peut précipiter ces jeunes dans des conduites suicidaires. Ces éléments nous ont poussés à faire de la divulgation de son homosexualité notre objet d'étude. Nous faisons l'hypothèse qu'au cours d'une annonce hautement stressante de leur homosexualité, les jeunes LG seraient particulièrement vulnérables et pourraient s'engager dans des conduites suicidaires. Nous souhaitions donc mieux comprendre cette situation stressante particulière et les liens qu'elle entretient avec les conduites et idéations suicidaires des jeunes LG. Pour mieux appréhender le stress présent lors de cette divulgation, nous nous sommes basés sur le «modèle transactionnel du stress » (Lazarus, 1966 ; Coyne et Lazarus, 1980 ; Lazarus et Folkman, 1984). Cette approche met l'accent sur la façon dont le sujet construit la transaction stressante. Elle considère que ce n'est pas l'événement qui est générateur de stress mais l'évaluation cognitive faite par l'individu. Le stress est donc présent lorsque la personne perçoit la situation comme dangereuse et qu'elle ne se sent pas capable d'y faire face. Différentes dimensions seraient donc impliquées dans la transaction stressante telles que l'évaluation cognitive de l'individu, les stratégies qu'il met en place pour gérer la situation ou encore son vécu émotionnel.

\section{METHODOLOGIE}

Les personnes interrogées ont toutes été rencontrées via l'internet dans différents sites de rencontre LGB (Gaypax, Gayvox). Des messages privés ont été envoyés aux profils des personnes habitant en région Provence-Alpes-Côte-d'Azur (PACA) (France). Ces messages précisaient la thématique de l'étude, le cadre de la recherche, ainsi que la procédure à suivre. Les personnes intéressées nous ont envoyé un courriel pour nous faire part de leur désir de participer à la recherche et ont été contactées par la suite. Un seul entretien semi-directif d'un durée d'environ une heure a été mené avec chaque participant (8 gays et 7 lesbiennes) par une 
seule et même chercheuse. Nous avons fait le choix de n'interroger que des personnes ayant entre 18 et 28 ans car c'est principalement au cours de cette période que les LGB commencent à annoncer leur homosexualité, mais également que leur risque suicidaire est le plus élevé (Dorais et Lajeunesse, 2001). Nous avons cherché à équilibrer le nombre de lesbiennes et de gays pour obtenir un échantillon homogène. Cette recherche a été réalisée sur un petit échantillon car il a été difficile de trouver des participants souhaitant participer et habitant en région PACA. Douze entretiens ont été réalisés en face à face dans des lieux publics et trois ont été menés par Skype, que ce soit pour une raison de distance géographique pour une jeune femme, ou par crainte de nous rencontrer en face à face pour deux jeunes hommes. Tous ont signé un formulaire de consentement avant l'entretien précisant notamment la confidentialité des informations et la possibilité d'arrêter l'entretien dès qu'ils le souhaitaient. Tous les entretiens ont été enregistrés à l'aide d'un dictaphone puis retranscrits et rendus anonymes.

Une grille d'entretien a été élaborée en lien avec nos objectifs de recherche. Elle est composée de deux thèmes : 1) l'annonce de leur homosexualité, l'annonce la plus stressante de leur vie et 2) les idées et les conduites suicidaires présentes au cours de leur vie. Chaque thème a été divisé en plusieurs sous-thèmes. Les sous-thèmes ont été élaborés en adéquation avec les dimensions du stress mises en avant dans le «modèle transactionnel » et visaient à appréhender les pensées, les émotions et les comportements des personnes interrogées, et ce, pour les deux thèmes. Tous les entretiens ont été menés sur la base de cette même grille pour tous les participants. En ce qui concerne le premier thème, ils ont été interrogés plus particulièrement sur le contexte dans lequel l'annonce de leur homosexualité a été la plus stressante, leurs attentes, leurs craintes ou encore les émotions ressenties et les comportements adoptés. En ce qui concerne le second thème, pour les personnes déclarant avoir eu des idées 
et/ou fait des tentatives de suicide au cours de leur vie, nous les avons questionnés sur le contexte de ces idées et/ou conduites suicidaires, ainsi que sur leurs motivations suicidaires.

À la fin de l'entretien, la chercheuse a demandé à chaque participant de commenter son vécu de l'échange et leur a laissé la possibilité d'ajouter quelque chose ou de poser des questions. Au vu des thèmes abordés lors de cette rencontre, elle leur a précisé qu'elle pouvait leur donner des coordonnées de thérapeutes s'ils en ressentaient le besoin, mais aucun jeune n'a répondu positivement. Les coordonnées de la chercheuse leur ont été laissées afin de leur permettre de la contacter en cas de besoin. Une fois les entretiens réalisés, une analyse de contenu du discours a été menée (Robert et Bouillaguet, 1997) dans le but de déterminer les thèmes qui sont apparus de manière récurrente au fil des entretiens. Suite à ces analyses, un pré-rapport a été transmis à l'ensemble des participants, énonçant les principaux résultats de la recherche. Les résultats présentés ici constituent une analyse exploratoire de ces entretiens, ils sont actuellement en train d'être analysés plus finement à l'aide d'un logiciel d'analyse lexicale. Par souci d'anonymat, les prénoms utilisés sont fictifs.

\section{RESULTATS}

\section{Thème 1 : l'annonce la plus stressante de leur homosexualité}

L'annonce la plus stressante de leur homosexualité a été réalisée en moyenne à 17,7 ans pour les jeunes hommes et à 19 ans pour les jeunes femmes. C'est majoritairement la divulgation auprès des parents qui s'est avérée la plus stressante (pour 13 sur 15) ou auprès d'amies ou d'amis lorsqu'elle constitue la première annonce (pour 2 sur 15) Elle s'est déroulée dans différents contextes :

- la découverte par l'entourage d'une lettre, d'un document ou d'un geste amoureux avec une personne du même sexe (3 jeunes) ; 
- l'écriture d'une lettre ou d'un courriel par le jeune pour dévoiler son orientation sexuelle à ses proches ( 4 jeunes $)$;

- $\quad$ une réponse à des questions de l'entourage (3 jeunes) ;

- $\quad$ une annonce verbale, en face à face, réalisée à l'initiative du jeune (4 jeunes) ;

- lors d'une tentative de suicide (1 jeune).

Nous pouvons constater que pour la majorité de nos participants l'annonce la plus stressante a été menée de manière indirecte et détournée. Les répondants qui ont pu décider du moment de leur annonce déclarent avoir été motivés par le besoin de sortir du secret, l'envie de ne plus mentir à des personnes aimées comme l'illustrent les extraits suivants : «je savais qu'un jour je devrais leur dire », « je ne pourrais pas me cacher toute ma vie », « on ne peut pas vivre éternellement dans le mensonge », « je ne voulais pas leur cacher, j'avais besoin de tout leur dire comme avant ».

Trois avantages à cette annonce ont été cités par nos participants. Tout d'abord, le fait de se libérer d'un poids, de ne plus avoir à mentir ou à se cacher comme l'illustrent les propos suivants : « Je ne voulais plus mentir (...) j'avais besoin qu'elle le sache pour m'en libérer »; « Le cacher, c'est un poids énorme, surtout que j'ai deux frères qui ont une copine et j'avais droit à des réflexions du genre "bon c'est quand que tu as une copine ?" et je ne savais pas quoi répondre, il faut le dire pour se sentir mieux, je pense, pour être "vrai" et ne pas avoir de “carapace"». Le désir de rompre avec leur sentiment de solitude et de bénéficier d'un soutien de leur part de leurs proches en partageant avec eux ce qu'ils étaient en train de vivre a également été évoqué par plusieurs jeunes : «J'étais perdue à cette période, ça a été une période très noire avec beaucoup de questions et j'avais besoin d'eux pour arriver à mieux comprendre les choses. » Pour d'autres cette révélation était un moyen de ne plus vivre avec 
la crainte perpétuelle d'être découvert : « Ma mère est très forte pour lire en moi et je savais qu'elle savait qu'il y avait un truc caché quelque part, alors que d'habitude je ne suis pas quelqu'un de secret, donc j'avais peur que ça tombe sans que je le contrôle. »

L'ensemble de nos répondants pensaient que cette révélation allait être difficile avant de la mener. Cette évaluation négative rendait donc la situation risquée et stressante. Trois principales craintes ont été mises en avant. Pour certains, leur principale crainte était d'être rejetés, d'être considérés comme différents et donc de perdre l'amour de leur proche : « Je me disais souvent : je suis pas normal, je suis un monstre, les autres vont me lâcher, ne plus me parler », « À chaque fois que j’y pensais, je m'imaginais le pire qu'ils me disent que je les dégoûte, qu'ils me rejettent ». Cette crainte est exacerbée chez les jeunes qui se sont confrontés à un rejet dans le passé : «j'avais peur de revivre ce que j’avais vécu avec ma meilleure amie.» Pour d'autres, le plus difficile était l'incertitude, l'inconnu, la difficulté à anticiper la réaction de leurs proches : «Je savais qu'elle avait rien contre les gays, mais son fils ,c'est différent, je ne savais pas comment elle pourrait réagir. » D'autres encore étaient dominés par la crainte d'apparaître comme une personne mauvaise, différente et anormale après avoir révélé son orientation sexuelle : «Ce qui bloque le plus, c'est d'avouer à quelqu'un qu'on aime qu'on est différent, parce qu'en fait être homo, ce n'est pas normal. Nous sommes tous conditionnés par le prince et la princesse dans les contes de fée, la maman et le papa. "

Les émotions qui précèdent l'annonce sont essentiellement des émotions dites « négatives ». Celles qui apparaissent le plus fréquemment dans le discours de nos répondantes et répondants sont tout d'abord la peur des réactions négatives, voire la panique : «Qu'est ce qu'il va dire? comment il va réagir? comment ça va se passer ? plein d'idées 
comme ça se bousculaient dans ma tête, je me suis jamais autant senti paniqué. » Le dégoût, essentiellement dirigé envers eux-mêmes est aussi de nombreuses fois évoqué : «Je me dégoûtais car pour moi, être homo, ce n'est pas un plaisir, c'est difficile à vivre chaque jour. » Et enfin, la gêne de se confronter à une situation inconnue : «J'étais gênée, je ne savais pas comment ça allait se passer, quoi lui dire. » Une fois l'annonce réalisée, le vécu émotionnel des répondants est très variable, et est étroitement lié à la réaction de l'entourage. La déception est l'émotion la plus fréquemment citée lorsque ces jeunes sont confrontés à des réactions négatives de la part de leurs proches: «Je me suis rendu compte qu'elle ne m’appréciait pas comme je suis réellement mais l'idée qu'elle se fait de moi », «J'ai eu l'impression d'être reniée, que je n'étais qu'un intérêt et une fierté pour ma mère, bonne à ramener des bonnes notes et à être sage mais surtout ne pas à salir son idéal ». Le soulagement et la libération sont des émotions qui sont mises en avant par beaucoup de jeunes, et ce, indépendamment de la réaction des proches. En effet, ces émotions se retrouvent à la fois chez des participants dont les parents ont mal réagi que chez ceux dont la réaction a été positive : «Après j'étais libéré, peu importe ce qu'ils pouvaient en penser, la vérité était le plus important, plus que le résultat, puisque j'étais prêt à mourir pour la vérité. » Et enfin la dernière émotion fréquemment évoquée au cours des entretiens est le bonheur, cette émotion n'étant présente que chez les jeunes qui ont considéré que leurs proches avaient bien réagi : «Quand elle m'a dit “tu fais ce que tu veux, ça changera rien”, des larmes de bonheur sont venues, le sourire aux lèvres et toute cette angoisse qui retombe en bloc, complètement vidé. » Il est important de noter qu'au fil du temps, le vécu émotionnel de ces jeunes a évolué généralement de manière positive, et ce, quelles qu'aient été les réactions premières de l'entourage : «J'ai eu un violent coup au cœur, un grand sentiment de déception, d'incompréhension, de rejet et de tristesse, puis j'ai rapidement relativisé, je me suis dis que ce n'était pas à elle de me juger que j'avais fait "ma part", que c'était son droit d'accepter ou 
non... Moi, j'étais à nouveau honnête avec moi-même, donc j'étais soulagé, j'avais juste des regrets par rapport à ce que je croyais être son ouverture d'esprit. »

Les comportements qu'ils mettent en place suite à l'annonce leur homosexualité sont étroitement liés à la manière dont ces jeunes ont préparé la divulgation de leur homosexualité et à la réaction de leur entourage. Les comportements sont très variés, il a donc été difficile de réaliser des regroupements. Certains ont adopté des stratégies de soutien, recherchant de l'aide et des conseils parmi leurs proches, ou encore des stratégies de résolution de problème, notamment en tentant de favoriser la communication : «J'ai essayé de discuter, mais elle n'a pas voulu m'adresser la parole et ça, pendant facilement une semaine. Et genre un mois plus tard, parce que je le vivais très mal, nous étions dans la voiture, pas le choix de me répondre, on s'est mis à discuter, elle a pleuré, elle s'est garée sur le bord de la route et on a parlé pendant quatre heures. » Cependant, d'autres ont adopté des stratégies plus dysfonctionnelles, notamment en s'engageant dans des comportements à risque (alcool, voiture...) ou des conduites auto-agressives (scarifications), en prononçant des propos violents, notamment dans le but de se venger: "J'ai essayé de la rendre honteuse de son comportement, j'ai été agressif. » D'autres encore ont essayé d'effacer ce qui s'était passé, comme par exemple en entretenant des relations amoureuses et/ou sexuelles avec des personnes du sexe opposé. Ces stratégies dysfonctionnelles ont essentiellement été employées par les plus jeunes et par ceux dont l'annonce a généré des réactions négatives.

\section{Thème 2 : idées et conduites suicidaires au cours de la vie}

Au sein de notre échantillon, 8 participants n'ont jamais pensé au suicide, 3 jeunes ont fait une tentative de suicide, 4 ont présenté des idées suicidaires sans passer à l'acte. Cette section 
est consacrée aux répondants ayant réalisé une tentative de suicide ou ayant eu des idéations suicidaires.

\section{Les conduites suicidaires}

Edwige a 18 ans lorsqu'elle annonce son homosexualité à ses parents. Cette divulgation est très mal accueillie et provoque de nombreuses tensions. Sa mère ne supportant plus de la voir la met à la porte. Elle se réfugie alors chez une amie chez qui elle vit durant six mois, rentrant occasionnellement chez ses parents certains soirs. Durant cette période, elle se met à consommer de l'alcool de manière abusive, à se scarifier, et fait plusieurs tentatives de suicide qui sont interrompues par l'intervention de son amie ou de sa famille. Lorsque Edwige évoque ses tentatives de suicide, elle précise avoir ressenti une réelle envie de mourir, ne percevant plus d'avenir à sa vie et se sentant honteuse de son orientation sexuelle. Selon elle, ses passages à l'acte étaient un moyen de protéger sa famille, de les préserver du mal qu'elle leur faisait en étant lesbienne : «Je devais me cacher, je devenais la honte de la famille, ma mère m'avait rejetée et je les rendais triste, mon frère souffrait. Je ne pouvais pas être moi et je ne voyais plus dans ce cas-là le sens de ma vie, pour moi, si je faisais souffrir et ne pouvais être moi, je préférais en arrêter là, je pensais à l'époque que ma mort aurait soulagé mes parents et mon frère du monstre que j'étais pour eux. » Mais au fil des mois, la mère d'Edwige commence peu à peu à revenir vers sa fille, l'amélioration des relations mère-fille ayant un impact important sur le bien-être d'Edwige qui précise avoir commencé à se sentir mieux lorsque sa mère s'est rapprochée d'elle.

Fabien dit avoir pris conscience de son homosexualité lorsqu'il avait 15 ans. Il a alors tenté de faire comprendre à ses parents qu'il était gay, de manière indirecte, mais sans y parvenir. Pour cela, il en parle autour de lui, à ses proches, aux proches de sa famille, dans 
l'espoir que cette information arrive jusqu'à ses parents, ne parvenant pas à leur en parler directement. Selon lui, cette situation l'a fait entrer peu à peu dans la dépression, ne supportant plus de garder ce secret. Au cours de cette période, son médecin lui prescrit un traitement antidépresseur qu'il associe à de fréquentes prises d'alcool. L'alcool lui permettait selon lui de gérer ses angoisses et lui donnait le «courage » d'annoncer son orientation sexuelle à son entourage. Cependant, il ne parvient toujours pas à le dire à ses parents, dominé par la peur de les blesser et d'être rejeté. À 17 ans, il prend la décision de leur écrire une lettre, leur précisant son envie de mourir, mais sans évoquer explicitement son homosexualité, et ingère une quantité importante de médicaments : «Je leur ai écrit que je voulais mourir, que je le faisais parce que je les aimais et, que s'ils voulaient avoir plus d'informations, ils devaient téléphoner à mon cousin. Je n'étais pas triste, j’étais libéré, et je les libérais, j'imaginais beaucoup mon enterrement, c'est bête, j'essayais de deviner qui viendrait, qui étaient les vrais amis. (...). Mon but était de me libérer et je ne trouvais rien d'autre comme solution, c'était la seule option possible pour moi à l'époque, je ne voulais pas rentrer dans un conflit et je ne supportais plus ce mensonge. » Lorsque ses parents le découvrent, ils le conduisent à l'hôpital et lui posent de nombreuses questions. Il finit par leur révéler son homosexualité, annonce que ses parents accueillent plutôt positivement.

Lorsqu'il a 18 ans, Bernard entame sa première relation amoureuse. Jusque là, il n'a jamais eu de relation. Il rencontre son premier compagnon sur l'internet, ils habitent dans des villes éloignées et ne se voient que de façon irrégulière. Après quelques mois de conversations sur l'internet, ils parviennent à se rencontrer et ont des relations sexuelles. Bernard précise être « devenu un homme ce jour-là ». Au bout d'un an, son compagnon met fin à leur relation, il ne supporte pas cette rupture et essaye de se pendre : «Mon monde s'est effondré, ça a duré un an, j'étais fou de lui, je ne pouvais pas le supporter. Pour moi, cette relation avait été une 
révélation... C'était stupide, j'ai pris une corde, mais ça n'a pas marché. Mon monde s'est écroulé, je n'en ai parlé à personne et j'ai essayé tant bien que mal de continuer malgré tout. » Bernard précise avoir mis plusieurs mois avant de parvenir à se remettre de cette relation et de ne plus penser à mettre fin à ses jours. Son premier compagnon avait été à la fois son premier amour, son premier partenaire sexuel et celui qui l'avait aidé à comprendre son orientation sexuelle. Il ajoute que ce qui a été le plus dur dans cette rupture, ça a été de gérer la solitude, perdant en même temps la seule personne avec qui il pouvait parler de son homosexualité. De plus, son entourage n'étant pas au courant de son orientation sexuelle, Bernard a eu beaucoup de difficultés à faire face seul à cette rupture, et a dû « donner le change », tentant de faire croire à son entourage que tout allait bien pour ne pas avoir à en parler et donc à révéler son homosexualité.

\section{Les idéations suicidaires}

À 17 ans, Jean n'a jamais divulgué son homosexualité à personne. Il appréhende ce moment qu'il repousse depuis plusieurs mois. Au cours de cette période, il dit avoir envisagé de nombreuses fois de mettre fin à ses jours, se sentant incapable de faire face au rejet qu'entraînerait cette annonce, et ne supportant plus la souffrance générée par ce secret : « J’y ai songé, mais je n'ai jamais eu le cran de passer à l'acte. Disons que je me disais constamment que c'était la porte de sortie ultime, si jamais ça n’allait pas. Mais je n'ai jamais sauté le pas, je suis toujours resté dans l'idée que ce serait la solution finale qui règlerait tous mes problèmes et qui mettrait fin à ma souffrance. » Il associe la divulgation de son homosexualité à de multiples craintes : «J'avais peur de me retrouver seul, incompris et jugé sur quelque chose d'infondé, j'avais également peur d'avoir des brimades de la part des autres à la sortie des cours. » Le suicide lui apparaissait alors comme un moyen d'éviter tout ça : « Ça m'aurait fait quitter ce monde qui me faisait tant souffrir à cause du rejet de mes amis. » 
Lors d'un repas, ses amis le poussent à dévoiler son homosexualité, en lui posant de nombreuses questions sur son orientation sexuelle. Il finit par le leur dire et reste étonné de constater qu'ils adoptent une attitude de soutien et rassurante. Selon Jean, si la réaction de ses amis n'avait pas été positive, il n'aurait pas eu les ressources pour affronter leur rejet, se sentant faible et non soutenu : «Personne chez moi n'aurait pu me soutenir si jamais j'avais été rejeté car personne n'était au courant de ma sexualité, je n'aurais pas pu le gérer.» À l'inverse, dès l'instant où ses amis ont été au courant, il s'est senti «plus fort», "plus confiant », pour faire face au rejet car il avait la conviction qu'il pourrait bénéficier de leur soutien.

Tatiana a commencé à ressentir une attirance envers une de ses amies à l'âge de 13 ans. Elle a alors beaucoup de difficultés à l'accepter : « Je me disais que ça n’était pas normal, je pensais être folle (...) j'étais perdue, complètement chamboulée, je me disais que jamais je n'aurais une vie heureuse à cause de cette "tendance". » Lorsqu'elle tente de dévoiler ses sentiments à son amie, elle se confronte à un rejet, ce qui vient accentuer son mal-être : « Cette fille ne m'aimait pas en retour, c'était très dur d'aimer autant sans retour. » Face à cette épreuve, elle précise s'être sentie très seule, n'ayant personne à qui en parler. Elle envisage alors de mettre fin à ses jours pour se libérer de ce mal-être : « J'avais envie de disparaître, c'était vraiment ça, je ne voulais plus être là pour ne pas avoir à affronter tout ça. C'était vraiment énorme pour moi, je n'ai jamais eu confiance en moi, alors ça, c'était trop, ça me paraissait insurmontable, c'est aussi à ce moment là que mes relations avec mes parents se sont dégradées, la fameuse crise d'ado ! ! Je ne sortais jamais de ma chambre, je ne parlais pas, où quand je leur parlais, je leur parlais mal. » Peu à peu, les autres élèves de son collège sont au courant, elle subit alors de nombreuses moqueries, mais bénéficie aussi du soutien de certains camarades. Dès lors, Tatiana se sent moins seule, ce qui l'aide à faire face aux 
attitudes négatives. Cependant, il lui a fallu plusieurs années avant d'accepter son homosexualité et de s'engager dans une relation amoureuse avec une femme.

Alizée a commencé à avoir des idées suicidaires à l'âge de 12 ans, et ce, durant environ deux ans. Elle met en lien ses idées noires avec le rejet qu'elle subissait de la part de ses camarades. Elle ajoute s'être sentie très différente à cette époque et avoir subi de nombreuses critiques de la part des autres élèves, critiques qui n'étaient pas directement liées à son homosexualité, car elle ajoute qu'elle-même à cette époque ne savait pas qu'elle était lesbienne : «Pendant le primaire et le collège, on n'a jamais cessé de me mettre à l'écart. C'est peut-être parce que j'avais une tenue vestimentaire pas terrible, et quoique... Je me suis souvent sentie différente. Je me sentais seule, j'étais toujours en conflit avec mes parents, j'avais une haine en moi et tous ces rejets n'ont fait qu'accentuer les choses. » Vers 14 ans, elle dit commencer à se sentir mieux, c'est également à cette époque qu'elle commence à se poser des questions sur son orientation sexuelle, et à entamer des discussions sur l'internet avec d'autres filles. Cependant elle ne fait pas spontanément de liens entre ces événements et l'amélioration de son humeur. Au fil des conversations, son homosexualité lui apparaît comme une évidence, ce qui constitue selon elle « une libération », elle pouvait enfin mettre un sens sur cette différence qu'elle avait toujours ressentie.

Lorsque Bertrand débute son collège, il est la cible de nombreux propos homophobes, subissant les moqueries des élèves de sa classe. Pourtant il précise qu'à cette époque, il ne savait pas qu'il était gay. Il se décrit comme un jeune homme ne correspondant pas aux stéréotypes de la masculinité, n'étant pas très sportif, plutôt mince et fréquentant essentiellement des filles. Souffrant de ces moqueries, il présente de nombreuses idées suicidaires durant toute sa scolarité : « Je voulais mourir pour en finir avec tout ça, j'en avais 
assez d'aller à l'école tous les jours avec la boule au ventre, et je ne pouvais en parler à personne, je ne voulais pas dire qu'on me traitait de pédé. » Il ajoute que lui-même avait une vision très négative des LGB, et il met de nombreuses années à prendre conscience de son orientation sexuelle et à l'accepter. Les propos homophobes qui lui ont été adressés durant des années ont pu avoir une incidence sur la construction de son identité sexuelle, reculant la prise de conscience de son homosexualité. Tout comme Alizée, dès l'instant où il a commencé à accepter son orientation sexuelle, il a également commencé à se «sentir mieux » et à «se sentir plus fort » pour affronter les moqueries des autres.

\section{DISCUSSION}

Parmi les 15 jeunes interrogés, deux jeunes hommes et une jeune femme ont réalisé une tentative de suicide. Pour deux jeunes, leur désir de mourir était lié à l'annonce de leur homosexualité, que ce soit par anticipation du rejet pour Fabien, ou suite à des réactions parentales négatives pour Edwige. Plus de la moitié des participants ont révélé leur homosexualité de manière indirecte (lettres, courriels...), en évitant la confrontation directe avec leurs proches. Ce comportement peut-être associé à leurs multiples craintes et à leur anticipation négative des réactions de leurs proches. Cette évaluation négative est un élément important pour mieux comprendre le stress ressenti par ces jeunes lors de cette annonce. Les

craintes évoquées rejoignent celles précisées par d'autres auteurs (Savin-Williams et Esterberg, 2000). De plus, les émotions négatives (peur, angoisse, gêne...) sont dominantes avant cette divulgation, et peuvent être mises en lien avec leur peur du rejet. Or, durant la divulgation de son homosexualité, la présence d'émotions négatives est corrélée au risque d'idéations et de conduites suicidaires, et cette présence d'émotions négatives est plus déterminante que l'absence d'émotions positives (Charbonnier et Graziani, 2012). 
Au sein de notre échantillon, l'annonce qui s'avère la plus stressante est celle réalisée auprès des parents et/ou la première. En effet, la première annonce est complexe, car comme le précise Jean, personne dans l'entourage n'est au courant et peut faire office de soutien. Le jeune se trouve alors seul à devoir affronter les éventuelles réactions négatives. Pour ce qui est des parents, de nombreux LGB n'annoncent pas leur homosexualité à leurs parents par peur que cette révélation ne modifie leurs relations avec eux (D’Augelli, Grossman et al., 2010). C'est notamment le cas de Fabien qui parvient à dévoiler son homosexualité à ses proches mais pas à ses parents, et en arrive à tenter de mettre fin à ses jours pour se libérer de ce secret qui devient insupportable pour lui. Dans le cas de Fabien, sa peur d'être rejeté pas ses parents ne s'est pas concrétisée, ses parents ayant réagi de manière plutôt positive. Cependant, dans certaines situations, les réactions parentales peuvent être très négatives et conduire certains jeunes à s'engager dans des conduites suicidaires, comme c'est le cas pour Edwige. Suite au rejet de ses parents, elle fait plusieurs tentatives de suicide, ne supportant plus d'avoir blessé ses proches et ne se supportant plus elle-même. Or, les adolescents se construisent en partie en fonction de la manière dont leurs parents les perçoivent ; l'acceptation ou le rejet parental peut donc avoir des conséquences négatives dans le développement des LGB et influer sur leur estime de soi (Rohner, 2004). Les LGB rejetés par leurs parents suite à l'annonce de leur homosexualité ont plus d'idées suicidaires que ceux qui sont acceptés (Ryan, Huebner et al., 2009). À l'inverse, l'acceptation et les liens familiaux forts rendent moins vulnérables les jeunes LGB qui auraient deux fois moins d'idées suicidaires que ceux qui sont rejetés (Eisenberg et Resnick, 2006).

Ce qui pousse majoritairement nos répondants à dévoiler leur homosexualité malgré la peur qu'ils ressentent, c'est leur désir de se libérer de ce secret, de ce mensonge. En effet, le maintien dans le secret leur est insupportable, comme c'est le cas pour Fabien. En ne 
dévoilant pas leur orientation sexuelle, certains LGB se retrouvent dans une position inconfortable et un état d'anxiété, dominés par la peur que leurs proches découvrent leur orientation sexuelle, et ce, jusqu'à qu'ils sortent du placard (Savin-Williams, 1998). Cette stratégie est donc coûteuse d'un point de vue cognitif, elle implique des processus cognitifs complexes, conscients et inconscients, qu'il faut mettre en place pour maintenir le secret tout en vivant dans l'appréhension d'être découvert (Smart et Wegner, 2000). Cependant, cette divulgation peut être associée à une profonde déception, essentiellement lorsque la réaction de l'entourage est négative. Or, la déception et l'anxiété à la suite de l'annonce de son homosexualité sont des émotions corrélées au risque de réaliser une tentative de suicide (Charbonnier et Graziani, 2012). Mais une fois cette divulgation faite, elle est majoritairement associée à un vécu émotionnel positif, elle constitue une expérience de libération. En effet, nos participants ont fréquemment évoqué un important «soulagement». Que ce soit pour Tatiana, qui a pu bénéficier du soutien de certains de ses camarades de classe une fois qu'elle a dévoilé son attirance pour une autre fille de sa classe. Ou pour Jean qui n'a plus présenté d'idées suicidaires après avoir annoncé son orientation sexuelle à ses amis, se sentant plus fort et plus soutenu. Ces données confirment donc que l'annonce de son homosexualité peut constituer une libération, un processus de « reconnaissance de soi » (Julien, 2000) favorisant l'épanouissement personnel (Coursaud, 2002). De plus, de nombreux auteurs ont mis en évidence les effets bénéfiques sur la santé mentale du soutien social même si celui-ci n'est que virtuel (Shaw et Gant, 2002 ; Tichon et Shapiro, 2003 ; Eastin et LaRose, 2005).

\section{CONCLUSION}

L'analyse thématique du discours de ces jeunes LG met en avant les liens existant entre l'annonce de leur homosexualité (ou son anticipation) et la présence d'idéations et de conduites suicidaires. Cependant, cette association varie selon les participants, elle peut-être 
associée à un rejet réel ou anticipé. De plus, il est important de préciser que la divulgation de son homosexualité est aussi une situation source de libération et de bien-être, essentiellement lorsqu'elle favorise la mise en place d'un soutien de la part de l'entourage. Le discours des jeunes interrogés démontre que d'autres contextes peuvent également être à risque, comme les déceptions amoureuses, la non-acceptation de son homosexualité ou encore le rejet des autres. Par conséquent, nous pouvons penser que les différentes dimensions de la construction de leur identité homosexuelle seraient probablement à risque (questionnements sur leur orientation sexuelle, non-acceptation de leur homosexualité, peur de révéler leur orientation sexuelle, rejet liés à leur orientation sexuelle, difficultés amoureuses...), ces risques étant majorés lors de la révélation de leur homosexualité. Notre faible effectif limite la portée de nos résultats et ne permet pas de les généraliser, mais cette recherche donne des pistes de réflexion sur le vécu de ces jeunes lors de l'annonce de leur homosexualité et sur les contextes où leur risque suicidaire est le plus marqué. Pour l'avenir, il serait intéressant de constituer un échantillon plus important et d'appréhender les différentes étapes de la construction de leur identité sexuelle, et non pas seulement celle de la divulgation de leur homosexualité. Ces données laissent à penser que, pour prévenir le risque suicidaire élevé des jeunes LG, il est essentiel de porter une attention particulière aux craintes que ces jeunes ont à l'égard de l'annonce de leur homosexualité et d'accompagner ceux qui se retrouvent en difficulté face à cette divulgation. Mais également, de mieux appréhender la construction de leur identité sexuelle et de leur vie amoureuse, et ce, afin d'être capable d'accompagner ceux qui seraient en souffrance durant ce processus et de prévenir leur risque suicidaire élevé.

\section{RÉFÉRENCES}

Adam, P. (2001). « Dépression, tentatives de suicide et prise de risque parmi les lecteurs de la presse gay française », Vulnérabilité des jeunes gays et lesbiennes et risque de suicide : État de la question et pistes de prévention. Synthèse de la journée d'étude 
organisée le 17 juillet 2001, Bruxelles, Observatoire du Sida et des Sexualités, Facultés universitaires Saint-Louis.

Bagley, C., et P. Tremblay (1997). " Suicidal behaviors in homosexual and bisexual males », Crisis, vol. 18, p. 24-34.

Bonet, L., B.E. Wells et J.T. Parsons (2007). «A positive look at a difficult time: A strength based examination of coming out for lesbian and bisexual women », Journal of LGBT Health Research, vol. 3, p. 7-14.

Bourdet, S., et J.M. Pugnière (2011). "Attirance sexuelle, suicidalité et homophobie intériorisée », dans D. Welzer-Lang et C. Z. Gaudron (dir.), Masculinité. État des lieux Toulouse, Erès, p. 113-123.

Charbonnier, E., et P. Graziani (2011). «La perception de jeunes lesbiennes et gais concernant l'attitude de leurs parents à l'égard de leur homosexualité », Revue canadienne de santé mentale communautaire, vol. 30, p. 31-46.

Charbonnier, E. et P. Graziani (2012). "Vécu émotionnel et tentatives de suicide lors du coming-out », La Revue Française et Francophone de Psychiatrie et de Psychologie Médicale, vol. 15, p. 24-29.

Clermont, M., et Y. Lacouture (2000). « Orientation sexuelle et santé », dans Institut de la statistique du Québec, Enquête sociale de la santé 1998, Québec, p. 219-230.

Coursaud, J.B. (2002). L'homosexualité entre préjugés et réalités, Toulouse, Milan.

Coyne, J.C., et R.S. Lazarus (1980). « Cognitive style, stress perception and coping », dans I. L. Kutasha et L. B. Schlesinger (dir.), Handbook of Stress and Anxiety: Knowledge, Theory, and Treatment, San Francisco, Jossey Bass, p. 144-158.

D’Augelli, A.R., A.H. Grossman, M.T. Starks et K.O. Sinclair (2010). « Factors associated with parents' knowledge of gay, lesbian, and bisexual youths' sexual orientation », Journal of GLBT family studies, vol. 6, p. 178-98.

D’Augelli, A.R. (2002). « Mental Health Problems among Lesbian, Gay, and Bisexual Youths Ages 14 to $21 »$, Clinical Child Psycholy and Psychiatry, vol. 7, p. 433-456

Dorais, M., et S.L. Lajeunesse (2001). Mort ou Fif. La face cache du suicide chez les garçons, Montréal, VLB éditeur.

Eastin, M.S., et R. LaRose (2005). "Alt.support: modelling social support online», Computers in Human Behavior, vol. 21, p. 977-992.

Eisenberg, M.E., et M.D. Resnick (2006). « Suicidality among gay, lesbian and bisexual youth: The role of protective factors ", Journal of adolescent health, vol. 39, p. 662668.

Halpin, S.A., et M. W. Allen (2004). " Changes in the psychosocial well-being during stages of gay identity development », Journal of Homosexuality, vol. 47, p. 109-126.

Iwasaki, Y., et J.L. Ristock (2007). "The nature of stress experienced by lesbians and gay men », Anxiety Stress Coping, vol. 20, p. 299-319.

Jouvin, E., D. Beaulieu-Prévost et D. Julien (2007). «Minorités sexuelles : des populations plus exposées que les autres ? » dans F. Beck, P. Guilbert et A. Gautier (dir.), Baromètre santé 2005, Paris, INPES, p. 355-367.

Julien, D. (2000). «Famille d'origine et homosexualité », dans M. Simard et J. Alary (dir.), Comprendre la famille : actes du $5^{e}$ symposium québécois de recherche sur la famille, Québec, Presses de l'Université du Québec, p. 210-222.

LaSala, M. (2000). "Gay male couples: The importance of coming out and being out to parents », Journal of Homosexuality, vol. 39, p. 47-71.

Lazarus, R.S. (1966). Psychological stress and coping process, New York, McGraw-Hill.

Lazarus, R.S., et S. Folkman (1984). Stress, Appraisal, and Coping, New York, Springer Publishing. 
Mathy, R.M. (2002). « Suicidality and sexual orientation in five continents: Asia, Australia, Europe, North America, and South America ». International Journal of Sexuality and Gender Studies, vol. 7, p. 215-225.

Meyer, I.H. (1995). « Minority stress and mental health in gay men », Journal of Health and Social Behavior, vol. 36, p. 38-56.

Meyer, I.H. (2003). " Prejudice, social stress, and mental health in lesbian, gay, and bisexual populations: conceptual issues and research evidence », Psychological Bulletin, vol. 129, p. 674-697.

Meyer, I.H., J. Dietrich et S. Schwartz (2008). « Lifetime prevalence of mental disorders and suicide attempts in diverse lesbian, gay, and bisexual populations », American Journal of Public Health, vol. 98, p. 1004-1006.

Meyer, I.H., et M.E. Northridge (2007). The Health of Sexual Minorities: Public Health Perspectives of Lesbian, Gay, Bisexual and Transgender Populations, New York, Springer.

Ministère de la Santé et des Services sociaux (1997). «L'adaptation des services sociaux et de santé aux réalités homosexuelles », Orientations ministérielles.

Ministère de la Santé et des Services sociaux (2010). «Plan d'action en santé mentale 20052010 : la force des liens $»$.

Monroe, E.J. (2001). «Drawing upon the experiences of those who are out: A qualitative study of the coming-out process of gays and lesbians ». Thèse de doctorat, Université de l'Iowa.

Morris, J.F. (1997). «Lesbian coming out as a multidimensional processes », Journal of Homosexuality, vol. 33, p. 1-22.

Otis, J., R. Noël, R. Lavoie, R. LeClerc, B. Turmel, M. Alary, R. Remis, J. Vincellette, R. Parent, M. Gaudreault, B. Mâsse et 1. g. d. r. Oméga (2002). «Événements suicidaires chez les hommes gais », Vis-à-vie, vol. 10, p. 15-17.

Padilla, Y.C., C. Crisp et D.L. Rew (2010). «Parental acceptance and illegal drug use among gay, lesbian, and bisexual adolescents: results from a National Survey », Social Work, vol. 55, p. 265-275.

Paul, J.P., J. Catania, L. Pollack, J. Moskowitz, J. Canchola, T. Mills, D. Binson et R. Stall (2002). " Suicide attempts among gay and bisexual men: lifetime prevalence and antecedents ». American Journal of Public Health, vol. 92, p. 1338-1345.

Remafedi, G. (2002). « Suicidality in a venue-based sample of young men who have sex with men », Journal of Adolescent Health, vol. 31, p. 305-310.

Robert, A., et A. Bouillaguet (1997). L'analyse de contenu, Paris, Presses universitaires de France.

Rohner, R.P. (2004). « The parental "acceptance-rejection syndrome": universal correlates of perceived rejection », American Psychologist, vol. 59, p. 830-840.

Rosario, M., E.W. Schrimshaw et J. Hunter (2004). "Gay-related stress and emotional distress among gay, lesbian, and bisexual youths: A longitudinal examination ». Journal of Consulting and Clinical Psychology, vol. 70, p. 967-975.

Ryan, C., D. Huebner, R.M. Diaz et J. Sanchez (2009). « Family rejection as a predictor of negative health outcomes in white and Latino lesbian, gay, and bisexual young adults "), Pediatrics, vol. 123, p. 346-52.

Saewyc, E.M., C.L. Skay, P. Hynds, S. Pettingell, L. H. Bearinger, M.D. Resnick et E. Reis (2007). «Suicidal ideation and attempts in North American school-based surveys: are bisexual youth at increasing risk? », Journal of LGBT Health Research, vol. 3, p. 2536. 
Savin-Williams, R.C. (1998). « The disclosure to families of same-sex attractions by lesbian, gay, and bisexual youths », Journal of Research on Adolescence, vol. 8, p. 49-68.

Savin-Williams, R. C. (2001). Mom, Dad, I'm gay: How families negotiate coming out, Washington (DC), American Psychological Association.

Savin-Williams, R.C., et K.G. Esterberg (2000). " Lesbian, gay, and bisexual families », dans D. H. Demo, K. R. Allen et M. A. Fine (dir.), Handbook of Family Diversity, New York, Oxford University Press, p. 197-215.

Shaw, L.H., et C. Gant (2002). «In defense of the Internet: the relationship between Internet communication and depression, loneliness, selfsteem and perceived social support », Cyber Psychology and Behavior, vol. 5, p. 157-171.

Silenzio, V., J. Peña, P. Duberstein, J. Cerel et K. Knox (2007). « Sexual orientation and risk factors for suicidal ideation and suicide attempts among adolescents and young adults », American Journal of Public Health, vol. 97, p. 2017-2019.

Smart, L., et D.M. Wegner (2000). « The hidden costs of stigma », dans T.F. Heatherton, R.E. Kleck, M.R. Hebl et J. G. Hull (dir.), The social psychology of stigma, New York, Guilford Press, p. 220-242.

Stevens, R.A. (2004). «Understanding gay identity development within the college environment ». Journal of College Student Development and Psychopathology, vol. 45, p. $185-206$.

Suicide Prevention Resource Center (2008). Suicide risk and prevention for lesbian, gay, bisexual, and transgender youth. M.A. Newton, Education Development Center Inc.

Tichon, J.G., et M. Shapiro (2003). " The process of sharing social support in cyberspace », Cyber Psychology and Behavior, vol. 6, p. 161-170.

Velter, A. (2007). Rapport Enquête Presse Gay 2004, Saint-Maurice ANRS, INVS.

Verdier, E., et J.M. Firdion (2003). Homosexualité et suicide. Etudes, témoignages et analyse. Montblanc, H\&O Éditions.

Willoughby, B.L.B., N.M. Malik et K.M. Lindahl (2006). «Parental Reactions to Their Sons' Sexual Orientation Disclosures: The Roles of Family Cohesion, Adaptability, and Parenting Style ». Psychology of Men \& Masculinity, vol. 7, p. 14-26.

Wong, C., et C.S. Tang (2004). "Coming out experiences and psychological distress of Chinese homosexual men in Hong Kong », Archives of Sexual Behavior, vol. 33, p. 149-157. 